\title{
Histomorphology of the stomach, proventriculus and ventriculus of the red jungle fowl.
}

\begin{abstract}
The cranial chamber (proventriculus) and caudal chamber (ventriculus) of the stomach of the Red jungle fowl (Gallus gallus spadiceus) were examined by means of light microscopy. Both chambers presented folds of the tunica mucosa lined by a simple prismatic epithelium that was positive for neutral mucin. Simple tubular glands occupied the lamina propria of both chambers; in the ventriculus of older birds, they showed a coiled base. These ventricular glands were lined by simple cuboidal cells represented by the chief cells and a few large basal cells. The luminal and tubular koilin rodlets and folds of the ventriculus were positive to periodic acid Schiff (PAS) stain. The proventricular glands were situated between the inner and outer layers of the lamina muscularis mucosae. Cells lining the tubulo-alveolar units of the proventricular glands showed a dentate appearance. Vacuoles were not observed, and the cells were negative for Alcian-PAS stain. The tunica submucosa was very thin in the proventricular wall. In the ventriculus, it was not separated from the lamina propria owing to the absence of any lamina muscularis mucosae. The tunica muscularis of the proventriculus was formed by a thick inner layer of circular smooth muscle fibres and a thin outer layer of longitudinal fibres. In addition to these layers, oblique muscle fibres formed the most internal layer of the tunica muscularis in the ventriculus.
\end{abstract}

Keyword: Histomorphology; Stomach; Proventriculus; Ventriculuc; Red jungle fowl. 